\title{
Targeted therapies for breast cancer
}

\author{
Michaela J. Higgins and José Baselga
}

\author{
Division of Hematology and Oncology, Massachusetts General Hospital, Boston, Massachusetts, USA.
}

\begin{abstract}
In recent years the description of well-defined molecular subtypes of breast cancer, together with the identification of the driving genetic alterations and signaling pathways, has led to the clinical development of a number of successful molecular targeted agents. This is best exemplified in the subset of HER2-amplified breast cancers, in which an increasing number of active agents are changing the natural history of this aggressive disease. Other targets are under exploration, and the clinical development of these agents will require a change from the current large, randomized trials in unselected patient populations to smaller trials in groups with a molecularly defined tumor type. In addition, combinatorial approaches that act on the secondary mutations and/or compensatory pathways in resistant tumors may markedly improve on the effects of targeted agents used alone.
\end{abstract}

\section{Introduction}

Breast cancer is a heterogeneous disease encompassing multiple subgroups with differing molecular signatures, prognoses, and responses to therapies (1). From the clinical view point, we can initially subdivide breast cancer into three major subtypes: tumors expressing estrogen receptors (ERs) and/or progesterone receptors (PRs) (commonly referred to as hormone receptor-positive [HR-positive] tumors), ERBB2-amplified (also known as human epidermal receptor 2-amplified [HER2-amplified]) breast cancer, and the remaining group commonly referred to as triple-negative breast cancer (TNBC) due to lack of expression of the ERs and PRs and normal or negative HER2 expression. This latter group itself encompasses a number of distinct entities with defined gene expression profiles and outcomes (1-3).

A greater understanding of the underlying biology of breast cancer has resulted in the identification of a number of molecular targets and development of novel therapeutics. Among them are tyrosine kinase inhibitors (TKIs) directed at a number of targets (HER1, HER2, HER3, IGF receptor [IGFR], C-MET, FGF receptor [FGFR]), inhibitors of intracellular signaling pathways (PI3K, AKT, mammalian target of rapamycin [mTOR], ERK), angiogenesis inhibitors, and agents that interfere with DNA repair. Some of these agents have shown remarkable activity and have already become part of the standard of care in patients with breast cancer (exemplified by the anti-HER2 agents trastuzumab and lapatinib). Others have shown clinical activity but are not yet approved for clinical practice. In this group are novel anti-HER2 agents as well as rapamycin analogs ("rapalogs," or inhibitors of mTOR) and the poly(ADP-ribose) polymerase (PARP) inhibitors for BRCA-deficient tumors (4-6). The third (and clearly the largest) group of compounds are still in an early phase of development, but in some cases, indications of clinical responses have already been observed.

One of the challenges going forward will be the ability to match each patient with the right therapy. For example, without exception, anti-HER2 therapies are only effective in tumors with HER2 amplification/overexpression. Similarly, only BRCA mutant tumors display exquisite sensitivity to PARP inhibitors as singleagent therapy (5). Recent observations in other tumor types, such as the high responses observed in $B-R A F$ mutant melanoma with $\mathrm{B}-\mathrm{RAF}$ inhibitors or with ALK inhibitors in lung cancers harboring $A L K$ mutations, provide support to the high rewards associated

Conflict of interest: The authors have declared that no conflict of interest exists. Citation for this article: J Clin Invest. 2011;121(10):3797-3803. doi:10.1172/JCI57152. with targeting therapies to tumors displaying certain mutations $(7,8)$. It is unclear whether we will find additional mutations that will correlate with a high level of drug sensitivity in breast cancer, but candidates include PI3K, AKT, and FGFR mutations as well as phosphatase and tensin homolog (PTEN) loss.

Other challenges to targeted therapies include acquired and primary resistance. Acquired resistance eventually develops in most patients in the advanced disease setting (9). Some mechanisms by which a tumor stops responding to a given therapy that it had initially responded to have been identified in HER2-positive tumors. These include loss of expression of the target as a result of continuous therapy (10), activation of mutations downstream from the target itself (11), and activation of additional mechanisms that promote cell proliferation (12). Primary resistance may occur due to lack of target dependency. In addition, it has been proposed recently that activation of compensatory pathways may rescue cells from the inhibitory effects of blocking just one target or pathway. For example, inhibition of the $\mathrm{PI} 3 \mathrm{~K} / \mathrm{AKT} / \mathrm{mTOR}$ pathway elicits compensatory activation of multiple survival routes including IGF1R and HER2, among others $(13,14)$. Combinatorial therapies that include clinical intervention at the level of these adaptive response mechanisms may improve outcomes, but distinct compensatory pathways are induced in a breast cancer cell type- and in a therapy-dependent context. Thus it is unlikely that a uniformly successful combinatorial approach to therapy will emerge.

In this review we will highlight some of the most promising targeted agents in development and discuss considerations for the optimal design of clinical trials of targeted therapies in breast cancer. In order to simplify the presentation, we will use the subclassification nomenclature currently used in the clinic. Targeted therapies currently available or in development for breast cancer subtypes are depicted in Figure 1.

\section{HR-positive breast cancer}

ER- and PR-positive breast cancer has, for more than three decades, been the prime example of cancer amenable to targeted drug approaches. Estrogen-focused therapies remain pivotal to the treatment of this disease, with the ER modulator tamoxifen improving survival among women with early and advanced breast cancer and further improvements provided by aromatase inhibitors (AIs) and the ER-degrading agent fulvestrant (15-18). Their long-term efficacy, however, is limited by relapse of disease and development of resistance. Despite continuous expression of ER at relapse in either locally recurrent or secondary metastatic tumors, 


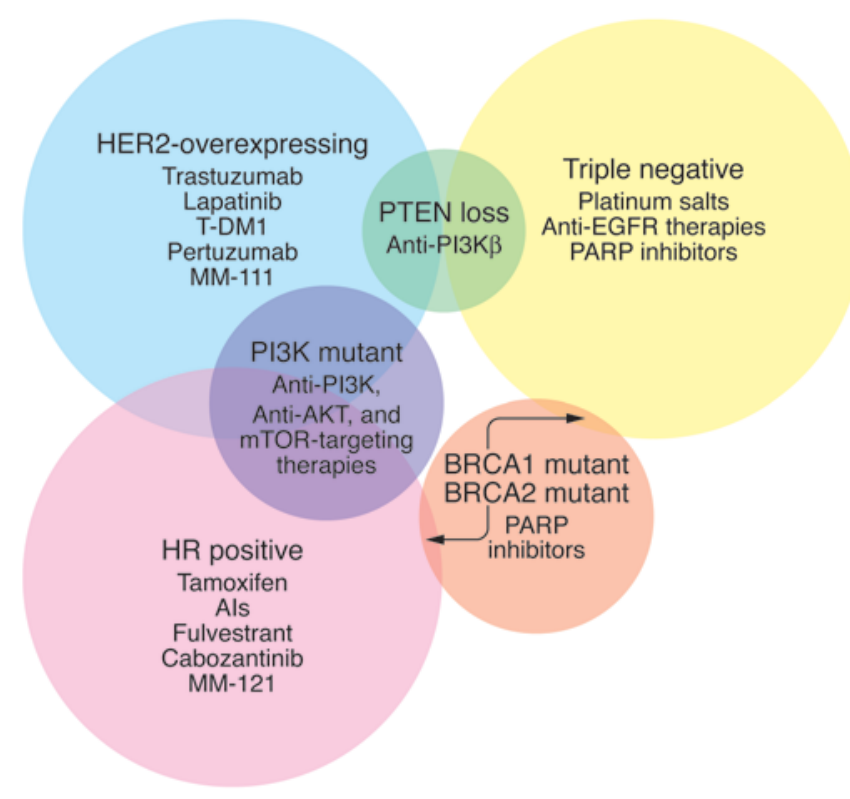

Figure 1

Venn diagram of breast cancer subtypes and their overlapping molecular targets. Selected targeted treatment strategies are also depicted.

up to $50 \%$ of patients with HR-positive primary breast cancer who develop metastatic disease do not respond to first-line endocrine treatment (de novo resistance), and the remainder will eventually relapse despite an initial response (acquired resistance) (19).

The HER family of proteins comprises 4 receptors (EGFR/HER1 and HER2-4) activated by numerous extracellular ligands. Upon ligand binding the receptors dimerize, become phosphorylated, and transduce intracellular signals that regulate a variety of cellular processes including proliferation and survival. Resistance to therapy can occur as a result of cross-talk between the ER and HER themselves or between signaling pathways downstream of these receptors, such as PI3K/Akt/mTOR (ref. 20 and Figure 2). HER2 overexpression confers intrinsic or primary resistance to hormonebased therapy despite the presence of HRs. Within tumors that are both HER 2 and ER positive, HER2 signaling is dominant, as demonstrated by the poor response of such tumors to endocrine therapy alone (21-24). This resistance can be partially overcome by combining anti-estrogen and anti-HER 2 therapies. The addition of the anti-HER 2 monoclonal antibody trastuzumab to an AI improved outcomes for patients with metastatic breast tumors that co-expressed both ER and HER2 (24). Likewise, in a large cohort of patients with known ER- and HER2-positive tumors, the addition of lapatinib, an anti-HER2 TKI, to an AI also significantly reduced the risk of progression (25). In contrast to HER2 and despite supportive preclinical data, observed clinical success with anti-HER 1 inhibitors and endocrine therapy combinations has been limited (26-28).

In addition to HER1 and HER2, there is growing interest in HER3 as a potential therapeutic target (29). Recently, HER3 and its physiologic ligand heregulin (HRG) have been implicated in the development of resistance to anti-estrogen therapies (30). There are now a number of anti-HER 3 monoclonal antibodies in development, including MM-121, a fully humanized monoclonal antibody that binds to HER3 and prevents the HRG- and betacel- lulin-induced phosphorylation of HER3 and also effectively inhibits the HER2/HER3 heterodimer (31). This compound is in Phase II studies, in combination with the nonsteroidal AI exemestane, in patients with advanced breast cancer that had previously progressed on endocrine therapies (32).

The PI3Ks phosphorylate the 3-hydroxyl group of phosphoinositides to activate second messenger molecules and set in motion a variety of physiological cellular metabolic and survival functions. Class IA PI3K molecules are heterodimers composed of a regulatory subunit (p85) and a catalytic subunit (p110), the $\alpha$ isoform of which is widely mutated or amplified in human cancer (33). The PI3K signaling pathway is critical for the growth and survival of cancer cells in many human tumors including breast (34-36). For this reason, multiple PI3K inhibitors are currently in clinical development (33). These agents display variable specificity to the different PI3K subunits and an ability to inhibit other targets such as mTOR. It is likely, but yet unproven, that these agents will be of most benefit in breast tumors harboring a somatic mutation in PIK3CA (the gene encoding $\mathrm{p} 110 \alpha$ ) or in those with nonfunctioning or absent PTEN protein. Although PIK3CA mutations are found in all breast cancer subtypes (at a frequency of approximately 30\%), they are most frequently identified in HR-positive or HER2-overexpressing tumors $(36,37)$. Although rarely mutated in breast cancer, diminished levels of PTEN expression through loss of heterozygosity and/or epigenetic silencing mechanisms are observed in up to $48 \%$ of breast tumors $(38,39)$. Interestingly, loss of PTEN has been shown to be more prevalent in triple-negative breast tumors and results in preferential activation of the PI3K $\beta$ subunit, an observation that suggests PI $3 \mathrm{~K} \beta$-specific inhibitors could be effective in this setting $(40,41)$. More advanced is the clinical development of rapalogs to inhibit mTOR. One of these agents, everolimus, has shown signs of improving the effects of AIs in a large presurgical study in patients with HR-positive breast cancer (42), and a randomized, placebo-controlled, Phase III study of everolimus in combination with exemestane is currently ongoing in the advanced disease setting (43).

As mentioned in the Introduction, inhibition of the PI3K/ $\mathrm{AKT} / \mathrm{mTOR}$ pathway elicits compensatory activation of multiple survival routes $(13,14)$. For example, it has been shown in tumors that inhibition of mTOR with rapalogs releases a negative feedback loop, resulting in activation of IGF1R signaling and ultimately phosphorylation of AKT (44). This activation may be prevented if IGF1R signaling is blocked with anti-IGF1R monoclonal antibodies. This finding led to a Phase I clinical study combining ridaforolimus (a rapalog) and dalotuzumab (an antibody targeted against IGF1R) that showed remarkable clinical activity in breast cancer (45). This combination is now being explored in a larger, Phase II study restricted to women with HR-positive metastatic breast cancer (46).

Almost a decade ago it was first suggested that anti-angiogenic strategies should be combined with drugs that target the proteins needed for cell motility and invasion, including hepatocyte growth factor (HGF) and C-MET (47), the rationale being that expression of these increases under hypoxic conditions and drives tumor cell survival and invasiveness even when anti-angiogenic agents are employed (48). Dual inhibition of MET and VEGFR2 would therefore be predicted to block major escape mechanisms used by tumors to overcome hypoxia. Cabozantinib (XL184; Exelixis) is a unique oral compound that inhibits multiple tyrosine kinases including MET and VEGFR2 (49). Preliminary data from an ongo- 


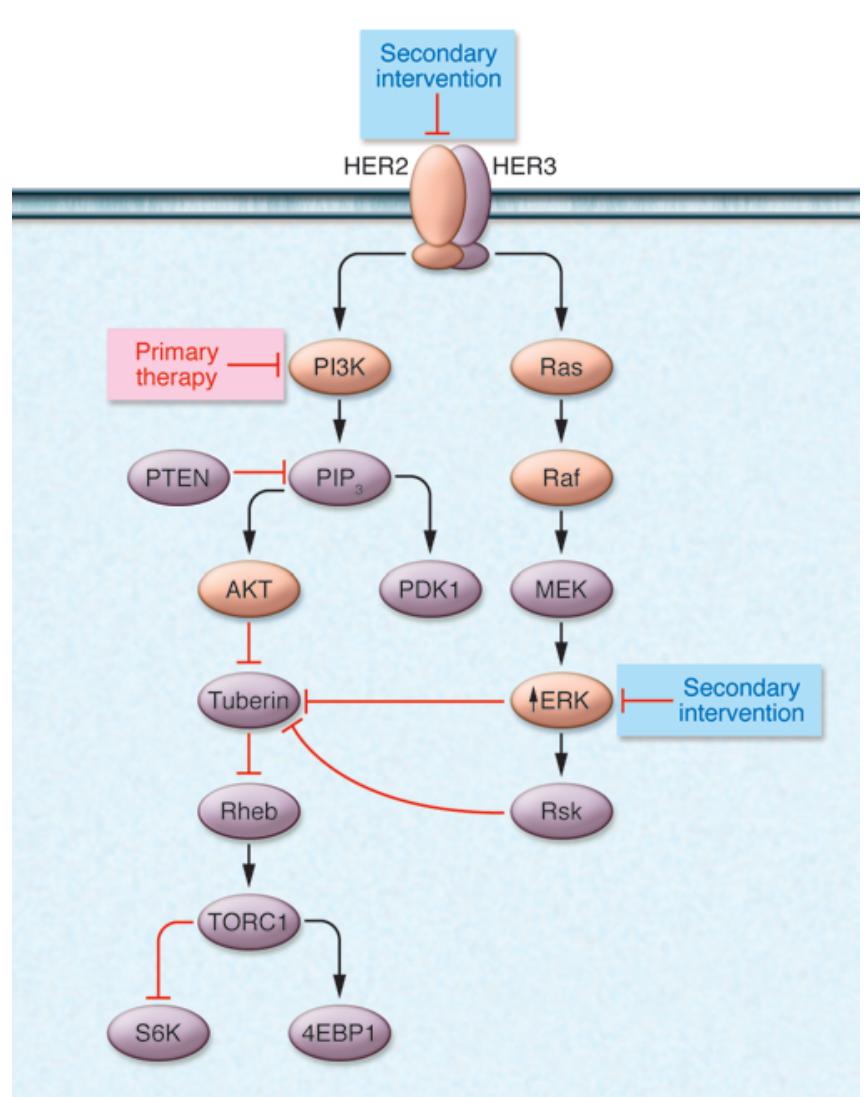

Figure 2

The activation of compensatory pathways may contribute to the development of resistance to targeted therapies in HER2-positive breast cancer. Inhibition of PI3K results in the release of a negative feedback loop and activation of HER2 and, in turn, activation of ERK, a potentially detrimental effect. Strategies to prevent the compensatory pathway include intervention at different levels, i.e., at the receptor level or by blocking ERK.

ing Phase II, randomized discontinuation trial of cabozantinib suggest remarkable activity of this agent in visceral sites of solid tumors but particularly within bone metastases, perhaps because HGF is expressed by both tumor cells and bone stroma, and MET is subsequently highly activated in bone metastases (50). A Phase II study of this agent is planned in patients with metastatic HR-positive breast cancer to bone.

\section{HER2-overexpressing tumors}

Less than a decade has passed since HER2 became an accepted therapeutic target in standard breast cancer practice. Trastuzumab in combination or sequence with cytotoxic chemotherapy transformed the prognosis of patients with HER2-overexpressing early breast cancer and is now routinely offered to this population mostly in combination with adjuvant chemotherapy $(51,52)$. Similarly, the dual HER1 and HER2 TKI lapatinib has clinical activity and is approved for the therapy of patients whose disease has progressed on trastuzumab (29). In addition to the approved agents, there are a number of novel strategies against HER2 that have shown activity in tumors that have progressed during treatment. One such approach has been the development of HER2-directed antibody-drug conjugates. Trastuzumab-DM1 (T-DM1) is an anti-HER2 antibody-drug conjugate consisting of trastuzumab covalently bound via a linker to DM1, a derivative of the antimicrotubule chemotherapy maytansine. T-DM1 uses trastuzumab to specifically localize the highly active chemotherapy to HER2-positive tumor cells. Upon binding to HER2, T-DM1 is internalized and undergoes proteolytic degradation, resulting in release of maytansine only inside of HER2-overexpressing cells (53). In a Phase I study of T-DM1 in patients with heavily pretreated, advanced HER2-positive breast cancer that had progressed during prior trastuzumab therapy, the clinical benefit rate among patients treated at the recommended dose was $73 \%$, including a number of objective responses (54). Single-agent activity was subsequently confirmed in a Phase II study among trastuzumab-resistant patients (55). Furthermore, preliminary results of a study of T-DM1 versus trastuzumab plus docetaxel in the firstline treatment of HER2-positive metastatic breast cancer patients suggest that T-DM1 provides similar efficacy to the combination with almost nonexistent side effects (56). It is now conceivable that we will develop systemic chemotherapy-free treatments for patients with HER2-amplified tumors.

Pertuzumab is a recombinant humanized monoclonal antibody directed against the dimerization domain II of HER2 that is required for ligand-dependent dimerization with HER3 (29). While trastuzumab prevents ligand-independent HER 2 signaling, pertuzumab interferes with ligand-dependent HER3-mediated signaling. In preclinical models, combined anti-HER2 blockade with trastuzumab and pertuzumab shows a synergistic effect in HER2-positive tumors (57). The combination of pertuzumab and trastuzumab among patients with metastatic HER2-positive breast cancer who had experienced progression during prior trastuzumab therapy demonstrated a clinical benefit rate of 50\% (4). Once again, a combination regimen that avoids the toxicity of systemic chemotherapy was highly active. The next logical question is whether T-DM1 and pertuzumab can be combined; thus an ongoing Phase III, randomized, three-arm, multicenter study will evaluate the efficacy and safety of T-DM1 plus pertuzumab or T-DM1 plus a placebo to pertuzumab, compared with the combination of trastuzumab plus taxane in patients with HER2-positive progressive or recurrent locally advanced or previously untreated metastatic breast cancer (58).

A similar approach is being studied with MM-111 (Merrimack Pharmaceuticals), a bispecific protein binding to HER2 and HER3 receptors, joined by a modified linker that inhibits HRG-induced activation of HER3 and AKT phosphorylation in dose-dependent manner. A Phase I/II trial of this compound in combination with trastuzumab is currently recruiting patients (59).

Resistance to anti-HER2 agents may occur as a result of aberrant activation of signalling pathways downstream of the receptor, such as the presence of activating PI3K mutations or loss of function of the phosphatase PTEN (refs. 11, 60, and Figure 2). In preclinical models, the addition of PI3K and/or mTOR inhibitors restores sensitivity to anti-HER2 agents (61). A recent Phase Ib study therefore combined the mTOR inhibitor everolimus with paclitaxel and trastuzumab in patients with HER2-overexpressing metastatic breast cancer pretreated with trastuzumab (62). Among the 27 patients evaluable for efficacy, the combination demonstrated encouraging antitumor activity with an overall response rate (ORR) of 44\%. Overall disease was controlled for at least six months in 74\% of those treated (62). HER2-targeting/PI3K inhibitor combinations are also in development. Another potential mechanism of resistance to HER2 monoclonal antibodies is the 
generation of truncated forms of the HER2 receptor that lack the trastuzumab-binding domain; tumors that harbor the truncated receptor may be resistant to antibody-based therapies and preferentially sensitive to kinase inhibitors (63).

\section{TNBC}

TNBC is an aggressive disease lacking a historical therapeutic target. In 2000, Perou et al. (2) first described distinct molecular subgroups of breast cancers based on variations in their gene expression profiles that correlated with prognosis; these subgroups included the basal epithelial-like group, an ERBB2-overexpressing group, a normal breast-like group and the ER-positive luminal A and $\mathrm{B}$ groups $(1,2)$. About $70 \%$ of breast tumors that are triple negative by immunohistochemistry cluster within the basal-like group of breast cancers on gene expression arrays (64). In addition to this overlap, a large majority of breast cancers arising in women with germline BRCA1 mutations have the triple-negative phenotype and also cluster among the basal-like group (65). It is now well established that inhibiting the remaining DNA repair machinery within BRCA-deficient cancer cells using PARP inhibition results in synthetic lethality (66). To prove this biologic principle in breast cancer patients, 54 subjects with $B R C A 1$ or BRCA2 mutations and advanced breast cancer were given the PARP inhibitor olaparib (AstraZeneca) (5). ORR was $41 \%$ among the 27 patients assigned to a 400-mg twice-daily dose without significant toxicity. These findings were complemented by the impressive clinical benefit, including a survival improvement, observed when another PARP inhibitor, iniparib (BSI-201; Sanofi), was combined with carboplatin and gemcitabine in a randomized Phase II trial among patients with TNBC (67). In late January 2011, the makers of iniparib (Sanofi) announced that the randomized Phase III trial evaluating iniparib in patients with metastatic TNBC (68) did not meet the prespecified criteria for significance for the coprimary endpoints of overall survival (OS) and progression-free survival (PFS), but the results of a prespecified analysis in patients who had received more than one prior line of chemotherapy for their metastic disease demonstrated an improvement in OS and PFS, consistent with what was seen in the Phase II study (69). These results make a strong case to better define the tumor population that may respond to this agent as well to PARP inhibitors in general. To this end, complementary biomarker studies are being performed in concert with many of the ongoing PARP inhibitor clinical trials, including pharmacogenetic analyses, testing of blood and tumor tissue for PARP activity and expression, and analysis of DNA repair enzyme status (70-72).

HER1 is also a potential target in TNBC. The anti-EGFR monoclonal antibody cetuximab has been shown to have limited activity as single agent in TNBC (73) but does increase the antitumor activity of platinum salts. In one randomized Phase II study, cetuximab given in combination with cisplatin resulted in a doubling of the response rate and improvement in time to disease progression and survival when compared with cisplatin alone (74). However, the performance of cisplatin as a single agent was disappointing, and it is not clear that the combination of cisplatin and cetuximab would compare favorably to conventional therapy in this disease setting. Nevertheless, these results cannot be ignored, as they point out that a subpopulation of TNBC may be sensitive to EGFR inhibition.

\section{Targeting angiogenesis in breast cancer}

The anti-VEGF-A monoclonal antibody bevacizumab was the first anti-angiogenic strategy to be rigorously evaluated in breast cancer, although additional anti-angiogenic compounds are now being studied. The landmark E2100 study randomized patients to receive the mitosis inhibitor paclitaxel alone or with bevacizumab and demonstrated increased response rates (21.2\% versus $36.9 \%$, respectively) and significantly prolonged PFS (5.9 versus 11.8 months, respectively) with the addition of bevacizumab (75). Among the HR-negative group of patients enrolled on this study (the large majority of whom were also HER2 negative), the median PFS was 4.6 months with paclitaxel and 8.8 months with paclitaxel plus bevacizumab (75). Subsequent studies with other chemotherapies demonstrated similar improvements in PFS but failed to show an OS advantage $(76,77)$. One possible reason for this may be trial design, which in the case of the RIBBON-1 and AVADO studies allowed for crossover to bevacizumab-containing therapy at the time of progression and may have masked an OS advantage $(76,77)$. It has also been demonstrated that potent antiangiogenic therapy over time can induce malignant progression of tumors due to increased invasiveness (78). Use of the bevacizumab has also been variably associated with an increased number of serious side effects, including stroke, wound healing complications and organ damage or failure (79). After careful review of the clinical data, the US FDA recommended that the breast cancer indication be removed from the bevacizumab label.

Sunitinib (Sutent; Pfizer) is a small-molecule multi-TKI that targets KIT, FLT3, RET, VEGFR2, and PDGFRB (80). In breast cancer, a single-agent Phase II study demonstrated clinical benefit in $16 \%$ of 64 heavily pretreated patients (81). However, two studies with sunitinib, either alone versus capecitabine or in combination with paclitaxel versus bevacizumab plus paclitaxel have been recently closed due to futility $(82,83)$. Sorafenib is another multikinase inhibitor targeting VEGFR1, VEGFR2, VEGFR3, PDGFRB, RAF, KIT, and FLT-3 (84). In a Phase II randomized trial in breast cancer patients, the addition of sorafenib to capecitabine significantly improved PFS compared with capecitabine alone (PFS 6.4 months versus 4.1 months) (85). The combination of capecitabine plus sorafenib is now being studied (versus capecitabine alone) in a large Phase III study (86).

The failure of bevacizumab to prolong OS has tempered expectations for the success of anti-angiogenic TKIs, but these compounds certainly have activity, and translational work to identify the subgroup of responsive patients will be critical. The lack of success of anti-angiogenic therapies to date in breast cancer may in part be explained by activation of additional pro-angiogenic switches upon blockade with bevacizumab, as has been shown in experimental systems (78).

\section{Design considerations for trials of targeted agents in breast cancer}

There are a number of challenges that still need to be addressed, such as the identification of biomarkers of response and early markers of clinical benefit. The study of mechanisms of resistance, as mentioned above, is also critically important. A frequent mechanism of primary resistance is lack of dependency on the targeted gene or pathway, such as the lack of activity of PARP inhibitors in breast tumors with intact BRCA function. This provides a strong argument for the development of early biomarkers of response and for the development of novel agents only in the subpopulation of breast tumors that may be dependent on the targeted gene. Acquired resistance, on the other hand, can be the result of acquired mutations that "overrule" the mechanism of action of 
the anticancer agent, such as mutations in the PI3K gene, downstream from HER2, which render cells insensitive to the effects of trastuzumab and lapatinib (87). This would require a combined therapeutic approach against the primary activating event (HER2 in this case) and the acquired mechanism of resistance (PI3K). There is an additional mechanism of resistance that relates to the activation of compensatory pathways that allow cells to "escape" the effects of therapeutic agents. Inhibition of certain molecular targets and/or pathways may result in activation of compensatory signaling pathways that prevent cell death. For example, AKT inhibition induces the expression and phosphorylation of multiple receptor tyrosine kinases (RTKs) (14). In a wide spectrum of tumor types, inhibition of AKT induces signaling via a conserved set of RTKs, including HER3, IGF1R, and insulin receptor; this may attenuate their antitumor activity. In this setting, combined inhibition of AKT and HER kinase activity is more effective than either alone (14). Similarly, inhibition of PI3K signaling results in activation of HER2 (13), which provides a strong rationale to block HER2 signaling in addition to PI3K.

Ongoing translational efforts should be focused on the development of standardized and validated biomarkers and functional imaging techniques that are able to indicate an early response (or lack thereof) to targeted agents in a variety of tumor types. These developments would ideally be tested in prospectively conducted, currently ongoing or completed clinical trials in which on-study tumor samples and other valuable materials including novel imaging approaches are carefully collected and annotated. Studies might also include patients with residual disease, in order to generate novel treatment approaches for this high-risk population. Such studies will require strong collaborations between clinical and laboratory researchers.

Once a biomarker has been identified, clinical trials of targeted agents may be performed within a specifically enriched patient population incorporating the predictive biomarker of clinical benefit. Study endpoints of clinical trials should therefore incorporate mechanistic effect measures on predefined markers and both tumor and stromal microenvironment. In addition, because compensatory pathways may be unknown and develop only during treatment with an experimental agent or combination of agents, efforts should be made to allow dynamic tuning of treatment selection within clinical studies. For example, trials would ideally incorporate a dynamic system of measurements of response or fail- ure to the study agent, which would allow real-time intrapatient change of treatment by either switching to another therapy or by adding a new agent. Such studies might explore collaboration with colleagues in functional imaging (e.g., PET, functional MRI) or use paired study tumor biopsies (baseline and on treatment) and circulating biomarkers in order to measure on-therapy effects. In breast cancer, the testing of new agents just prior to surgery (known as neo-adjuvant therapy) allows for monitoring of tumor response at the time of surgical resection. In comparison with the follow-up period of at least five years that is traditionally required to evaluate the efficacy of an adjuvant therapy, the neo-adjuvant setting enables the rapid assessment of tumor sensitivity within three to four months at most $(42,88-90)$. A further advantage is the easy availability of tumor tissue samples during the treatment period. A potential disadvantage of neo-adjuvant therapy in general is the loss of prognostic information provided by tumor size and nodal status at surgery and before adjuvant chemotherapy (91). These designs rely upon surrogate markers (such as changes in proliferation or apoptosis markers, or even absence of visible tumor at the time of surgery) that will then be correlated with endpoints of clinical benefit (such as time free of disease or improved OS).

\section{Conclusions}

Current treatment options for breast cancer are moving toward nontoxic, potent targeted therapies that can be tailored to an individual patient's tumor. There are now targeted therapeutic options available for nearly all breast cancer subtypes, exploiting the differing drivers of carcinogenesis within these individual tumors. The continuing development, and indeed success, of these compounds will rely heavily on close collaborations between laboratory scientists and clinician researchers. The development of resistance to all of these therapies is an ongoing challenge and opportunity for learning. In concert with robust clinical trials of these agents, biomarkers of response or prediction of benefit to these interventions must be developed and validated. Just as cancer is a dynamic, adaptive process, so too must our clinical trial designs become innovative, flexible, and informative. In this way we will select the right patient, for the best drug or combinations of drugs, at the most effective time.

Address correspondence to: José Baselga, Hematology/Oncology, 55 Fruit Street, YAW 9, Boston, Massachusetts 02114-2696, USA. Phone: 617.724.4800; Fax: 617.643.2683; E-mail: jbaselga@ partners.org.
1. Sorlie T, et al. Gene expression patterns of breast carcinomas distinguish tumor subclasses with clinical implications. Proc Natl Acad Sci U S A. 2001; 98(19):10869-10874.

2. Perou CM, et al. Molecular portraits of human breast tumours. Nature. 2000;406(6797):747-752.

3. Carey LA, et al. Race, breast cancer subtypes, and survival in the carolina breast cancer study. JAMA. 2006;295(21):2492-2502.

4. Baselga J, et al. Phase II trial of pertuzumab and trastuzumab in patients with human epidermal growth factor receptor 2-positive metastatic breast cancer that progressed during prior trastuzumab therapy. J Clin Oncol. 2010;28(7):1138-1144.

5. Tutt A, et al. Oral poly(ADP-ribose) polymerase inhibitor olaparib in patients with BRCA1 or BRCA2 mutations and advanced breast cancer: a proof-ofconcept trial. Lancet. 2010;376(9737):235-244.

6. Macaskill EJ, et al. The mammalian target of rapamycin inhibitor everolimus (RAD001) in early breast cancer: results of a pre-operative study. Breast Cancer Res Treat. 2011;128(3):725-734.
7. Flaherty KT, et al. Inhibition of mutated, activated BRAF in metastatic melanoma. $N$ Engl J Med. 2010;363(9):809-819.

8. Kwak EL, et al. Anaplastic lymphoma kinase inhibition in non-small-cell lung cancer. $N$ Engl J Med. 2010;363(18):1693-1703.

9. Wong ST, Goodin S. Overcoming drug resistance in patients with metastatic breast cancer. Pharmacotherapy. 2009;29(8):954-965.

10. Mittendorf EA, et al. Loss of HER2 amplification following trastuzumab-based neoadjuvant systemic therapy and survival outcomes. Clin Cancer Res. 2009;15(23):7381-7388.

11. Berns $\mathrm{K}$, et al. A functional genetic approach identifies the PI3K pathway as a major determinant of trastuzumab resistance in breast cancer. Cancer Cell. 2007;12(4):395-402.

12. Scaltriti M, et al. Cyclin E amplification/overexpression is a mechanism of trastuzumab resistance in HER2+ breast cancer patients. Proc Natl Acad Sci US A. 2011;108(9):3761-3766.

13. Serra V, et al. PI3K inhibition results in enhanced
HER signaling and acquired ERK dependency in HER2-overexpressing breast cancer. Oncogene. 2011;30(22):2547-2557.

14. Chandarlapaty S, et al. AKT inhibition relieves feedback suppression of receptor tyrosine kinase expression and activity. Cancer Cell. 2011;19(1):58-71.

15. Early Breast Cancer Trialists' Collaborative Group (EBCTCG). Effects of chemotherapy and hormonal therapy for early breast cancer on recurrence and 15-year survival: an overview of the randomised trials. Lancet. 2005;365(9472):1687-1717.

16. Gradishar WJ. Adjuvant endocrine therapy for early breast cancer: the story so far. Cancer Invest. 2010;28(4):433-442.

17. Gibson L, Lawrence D, Dawson C, Bliss J. Aromatase inhibitors for treatment of advanced breast cancer in postmenopausal women. Cochrane Database Syst Rev. 2009;(4):CD003370.

18. Robertson JF, et al. Activity of fulvestrant $500 \mathrm{mg}$ versus anastrozole $1 \mathrm{mg}$ as first-line treatment for advanced breast cancer: results from the FIRST study. J Clin Oncol. 2009;27(27):4530-4535. 
19. Ring A, Dowsett M. Mechanisms of tamoxifen resistance. Endocr Relat Cancer. 2004;11(4):643-658.

20. Prat A, Baselga J. The role of hormonal therapy in the management of hormonal-receptor-positive breast cancer with co-expression of HER2. Nat Clin Pract Oncol. 2008;5(9):531-542.

21. Lipton A, et al. Elevated serum Her-2/neu level predicts decreased response to hormone therapy in metastatic breast cancer. J Clin Oncol. 2002; 20(6):1467-1472.

22. Dowsett $M$, et al. Relationship between quantitative estrogen and progesterone receptor expression and human epidermal growth factor receptor 2 (HER-2) status with recurrence in the Arimidex, Tamoxifen, Alone or in Combination trial. J Clin Oncol. 2008;26(7):1059-1065.

23. De Laurentiis M, et al. A meta-analysis on the interaction between HER-2 expression and response to endocrine treatment in advanced breast cancer. Clin Cancer Res. 2005;11(13):4741-4748.

24. Kaufman B, et al. Trastuzumab plus anastrozole versus anastrozole alone for the treatment of postmenopausal women with human epidermal growth factor receptor 2-positive, hormone receptor-positive metastatic breast cancer: results from the randomized phase III TAnDEM study. J Clin Oncol. 2009;27(33):5529-5537

25. Johnston S, et al. Lapatinib combined with letrozole versus letrozole and placebo as first-line therapy for postmenopausal hormone receptorpositive metastatic breast cancer. J Clin Oncol. 2009; 27(33):5538-5546

26. Osborne CK, et al. Gefitinib or placebo in combination with tamoxifen in patients with hormone receptor-positive metastatic breast cancer: a randomized phase II study. Clin Cancer Res. 2011; 17(5):1147-1159

27. Cristofanilli $\mathrm{M}$, et al. Phase II, randomized trial to compare anastrozole combined with gefitinib or placebo in postmenopausal women with hormone receptor-positive metastatic breast cancer. Clin Cancer Res. 2010;16(6):1904-1914.

28. Baselga J, et al. Phase II and tumor pharmacodynamic study of gefitinib in patients with advanced breast cancer. J Clin Oncol. 2005;23(23):5323-5333.

29. Baselga J, Swain SM. Novel anticancer targets: revisiting ERBB2 and discovering ERBB3. Nat Rev Cancer. 2009;9(7):463-475.

30. Frogne T, et al. Activation of ErbB3, EGFR and Erk is essential for growth of human breast cancer cell lines with acquired resistance to fulvestrant. Breast Cancer Res Treat. 2009;114(2):263-275.

31. Schoeberl B, et al. An ErbB3 antibody, MM-121, is active in cancers with ligand-dependent activation. Cancer Res. 2010;70(6):2485-2494.

32. Merrimack. Trial of exemestane +/- MM-121 in postmenopausal women with locally advanced or metastatic estrogen receptor positive and/or progesterone receptor positive her 2 negative breast cancer. NIH Web site. http://clinicaltrials.gov/ct2/show/ results/NCT01151046. Accessed June 10, 2011.

33. Markman B, Atzori F, Perez-Garcia J, Tabernero J, Baselga J. Status of PI3K inhibition and biomarker development in cancer therapeutics. Ann Oncol. 2010;21(4):683-691.

34. Karakas B, Bachman KE, Park BH. Mutation of the PIK3CA oncogene in human cancers. Br J Cancer. 2006;94(4):455-459.

35. Bachman KE, et al. The PIK3CA gene is mutated with high frequency in human breast cancers. Cancer Biol Ther. 2004;3(8):772-775.

36. Saal LH, et al. PIK3CA mutations correlate with hormone receptors, node metastasis, and ERBB2, and are mutually exclusive with PTEN loss in human breast carcinoma. Cancer Res. 2005;65(7):2554-2559.

37. Stemke-Hale K, et al. An integrative genomic and proteomic analysis of PIK3CA, PTEN, and AKT mutations in breast cancer. Cancer Res. 2008 ;
68(15):6084-6091.

38. Perren A, et al. Immunohistochemical evidence of loss of PTEN expression in primary ductal adenocarcinomas of the breast. Am J Pathol. 1999; 155(4):1253-1260

39. Depowski PL, Rosenthal SI, Ross JS. Loss of expression of the PTEN gene protein product is associated with poor outcome in breast cancer. Mod Pathol. 2001;14(7):672-676.

40. Berrada N, Delaloge S, Andre F. Treatment of triple-negative metastatic breast cancer: toward individualized targeted treatments or chemosensitization? Ann Oncol. 2010;21(suppl 7):vii30-vii35.

41. Ilic N, Roberts TM. Comparing the roles of the p110alpha and p110beta isoforms of PI3K in signaling and cancer. Curr Top Microbiol Immunol. 2010; 347:55-77.

42. Baselga J, et al. Phase II randomized study of neoadjuvant everolimus plus letrozole compared with placebo plus letrozole in patients with estrogen receptor-positive breast cancer. J Clin Oncol. 2009; 27(16):2630-2637.

43. Novartis. Everolimus in combination with exemestane in the treatment of postmenopausal women with estrogen receptor positive locally advanced or metastatic breast cancer who are refractory to letrozole or anastrozole (BOLERO-2). NIH Web site. http://clinicaltrials.gov/ct2/show/ NCT00863655?term=NCT00863655\&rank=1. Accessed June 10, 2011

44. Tabernero J, et al. Dose- and schedule-dependent inhibition of the mammalian target of rapamycin pathway with everolimus: a phase I tumor pharmacodynamic study in patients with advanced solid tumors. J Clin Oncol. 2008;26(10):1603-1610.

45. Di Cosimo S, et al. A phase I study of the oral mTOR inhibitor ridaforolimus (RIDA) in combination with the IGF-1R antibody dalotozumab (DALO) in patients (pts) with advanced solid tumors. J Clin Oncol. 2010;28(18):Abstr 3008

46. Merck. A study of ridaforolimus (MK-8669) in combination with dalotuzumab (MK-0646) compared to standard of care treatment in estrogen receptor positive breast cancer patients (MK-8669041). NIH Web site. http://clinicaltrials.gov/ct2/ show/NCT01234857?term=NCT01234857\& rank=1. Accessed June 10, 2011

47. Bottaro DP, Liotta LA. Cancer: Out of air is not out of action. Nature. 2003;423(6940):593-595.

48. Pennacchietti S, Michieli P, Galluzzo M, Mazzone M, Giordano S, Comoglio PM. Hypoxia promotes invasive growth by transcriptional activation of the met protooncogene. Cancer Cell. 2003;3(4):347-361.

49. Durante C, Russo D, Verrienti A, Filetti S. XL184 (cabozantinib) for medullary thyroid carcinoma. Expert Opin Investig Drugs. 2011;20(3):407-413.

50. Exelixis. Study of cabozantinib (XL184) in adults with advanced malignancies. NIH Web site. http:// clinicaltrials.gov/ct2/show/NCT00940225?term= NCT00940225\&rank=1. Accessed June 10, 2011.

51. Piccart-Gebhart MJ, et al. Trastuzumab after Adjuvant Chemotherapy in HER2-Positive Breast Cancer. N Engl J Med. 2005;353(16):1659-1672.

52. Romond EH, et al. Trastuzumab plus Adjuvant Chemotherapy for Operable HER2-Positive Breast Cancer. NEngl J Med. 2005;353(16):1673-1684.

53. Lewis Phillips GD, et al. Targeting HER2-positive breast cancer with trastuzumab-DM1, an antibody-cytotoxic drug conjugate. Cancer Res. 2008; 68(22):9280-9290.

54. Krop IE, et al. Phase I study of trastuzumab-DM1, an HER2 antibody-drug conjugate, given every 3 weeks to patients with HER2-positive metastatic breast cancer. J Clin Oncol. 2010;28(16):2698-2704.

55. Burris HA 3rd, et al. Phase II study of the antibody drug conjugate trastuzumab-DM1 for the treatment of human epidermal growth factor receptor 2 (HER2)-positive breast cancer after prior HER2- directed therapy. J Clin Oncol. 2011;29(4):398-405.

56. Perez EA, et al. Efficacy and safety of trastuzumabDM1 versus trastuzumab plus Docetaxel in HER2positive metastatic breast cancer patients with no prior chemotherapy for metastatic disease: preliminary results of a randomized, multicenter, openlabel phase 2 study. Presented at: European Society of Medical Oncology; May 2010; Milan, Italy.

57. Scheuer W, Friess T, Burtscher H, Bossenmaier B, Endl J, Hasmann M. Strongly enhanced antitumor activity of trastuzumab and pertuzumab combination treatment on HER2-positive human xenograft tumor models. Cancer Res. 2009;69(24):9330-9336.

58. Hoffmann-La Roche. A study of trastuzumab$\mathrm{dm} 1$ plus pertuzumab versus trastuzumab [Herceptin] plus a taxane in patients with metastatic breast cancer (MARIANNE). NIH Web site. http:// clinicaltrials.gov/ct2/show/NCT01120184?term= NCT01120184\&rank=1. Accessed June 13, 2011.

59. Merrimack. A study of MM-111 in combination with (Herceptin) trastuzumab in patients with advanced Her2 positive breast cancer. NIH Web site. http://clinicaltrials.gov/ct2/show/ NCT01097460? term=NCT01097460\&rank $=1$. Accessed June 13, 2011.

60. Eichhorn PJ, et al. Phosphatidylinositol 3-kinase hyperactivation results in lapatinib resistance that is reversed by the mTOR/phosphatidylinositol 3-kinase inhibitor NVP-BEZ235. Cancer Res. 2008;68(22):9221-9230.

61. Serra V, et al. NVP-BEZ235, a dual PI3K/mTOR inhibitor, prevents $\mathrm{PI} 3 \mathrm{~K}$ signaling and inhibits the growth of cancer cells with activating PI3K mutations. Cancer Res. 2008;68(19):8022-8030.

62. Andre F, et al. Phase I study of everolimus plus weekly paclitaxel and trastuzumab in patients with metastatic breast cancer pretreated with trastuzumab. J Clin Oncol. 2010;28(34):5110-5115.

63. Scaltriti M, et al. Expression of p95HER2, a truncated form of the HER2 receptor, and response to anti-HER2 therapies in breast cancer. J Natl Cancer Inst. 2007;99(8):628-638.

64. Irvin WJ Jr, Carey LA. What is triple-negative breast cancer? Eur J Cancer. 2008;44(18):2799-2805.

65. Collins LC, et al. Basal cytokeratin and epidermal growth factor receptor expression are not predictive of BRCA1 mutation status in women with triple-negative breast cancers. Am J Surg Pathol. 2009; 33(7):1093-1097.

66. Iglehart JD, Silver DP. Synthetic lethality--a new direction in cancer-drug development. $N$ Engl J Med. 2009;361(2):189-191.

67. O'Shaughnessy J, et al. Iniparib plus chemotherapy in metastatic triple-negative breast cancer. $N$ EnglJ Med. 2011;364(3):205-214.

68. Sanofi-Aventis. A phase 3, multi-center study of gemcitabine/carboplatin, with or without BSI-201, in patients with ER-, PR-, and Her2-negative metastatic breast cancer. NIH Web site. http://clinicaltrials. gov/ct2/show/NCT00938652?term=NCT00938652 \&rank=1. Accessed June 13, 2011

69. Sanofi-Aventis. Positive phase II results with BSI201 (Iniparib) in women with metastatic triple negative breast cancer [press release]. Paris, France: Associated Press; January 5, 2011.

70. Cancer Research UK. AG014699 in treating patients with locally advanced or metastatic breast cancer or advanced ovarian cancer. NIH Web site. http:// clinicaltrials.gov/ct2/show/NCT00664781?term= NCT +00664781 \&rank=1. Accessed June 13, 2011.

71. Hoosier Oncology Group. PARP inhibition for triple negative breast cancer (ER-/PR-/HER2-) with BRCA1/2 mutations. NIH Web site. http:// clinicaltrials.gov/ct2/show/NCT01074970?term= NCT+01074970\&rank=1. Accessed June 13, 2011.

72. Massachusetts General Hospital. ABT-888 and temozolomide for metastatic breast cancer and BRCA1/2 breast cancer. NIH Web site. http://clinicaltrials. 
gov/ct2/show/NCT01009788?term=NCT01009788 \&rank=1. Accessed June 13, 2011.

73. Carey LA, et al. TBCRC 001: EGFR inhibition with cetuximab added to carboplatin in metastatic triple-negative (basal-like) breast cancer. J Clin Oncol. 2008;26:(suppl):Abstr 1009.

74. Baselga J, et al. The addition of cetuximab to cisplatin increases overall response rate and progression free survival in metastatic triple-negative breast cancer: results of a randomized phase II study (BALI-1). Ann Oncol. 2010;21(suppl 7):viii96-viii121.

75. Miller K, et al. Paclitaxel plus bevacizumab versus paclitaxel alone for metastatic breast cancer. $N$ Engl JMed. 2007;357(26):2666-2676.

76. O'Shaughnessy JA, Brufsky AM. RiBBON 1 and RiBBON 2: phase III trials of bevacizumab with standard chemotherapy for metastatic breast cancer. Clin Breast Cancer. 2008;8(4):370-373.

77. Miles DW, et al. Phase III study of bevacizumab plus docetaxel compared with placebo plus docetaxel for the first-line treatment of human epidermal growth factor receptor 2-negative metastatic breast cancer. J Clin Oncol. 2010;28(20):3239-3247.

78. Paez-Ribes M, et al. Antiangiogenic therapy elicits malignant progression of tumors to increased local invasion and distant metastasis. Cancer Cell. 2009; 15(3):220-231.

79. Choueiri TK, et al. Congestive heart failure risk in patients with breast cancer treated with bevacizumab. JClin Oncol. 2011;29(6):632-638.

80. Deprimo S, et al. Effect of treatment with suni- tinib malate, a multitargeted tyrosine kinase inhibitor, on circulating plasma levels of VEGF, soluble VEGF receptors 2 and 3 , and soluble KIT in patients with metastatic breast cancer. J Clin Oncol. 2006;24(18S):578.

81. Miller K, et al. Phase II study of SU11248, a multitargeted receptor tyrosine kinase inhibitor (TKI), in patients (pts) with previously treated metastatic breast cancer (MBC). J Clin Oncol. 2005; 23(16S):563.

82. Pfizer. Study of sunitinib with capecitabine in breast cancer. NIH Web site. http://clinicaltrials.gov/ ct $2 /$ show $/$ NCT00662025?term=NCT00662025 \&rank=1. Accessed June 13, 2011.

83. Pfizer. A study of SU011248 plus paclitaxel versus bevacizumab plus paclitaxel in patients with advanced breast cancer. NIH Web site. http:// clinicaltrials.gov/ct2/show/NCT00373256?term= NCT003732568rank=1. Accessed June 13, 2011.

84. Wilhelm SM, et al. BAY 43-9006 Exhibits broad spectrum oral antitumor activity and targets the $\mathrm{RAF} / \mathrm{MEK} / \mathrm{ERK}$ pathway and receptor tyrosine kinases involved in tumor progression and angiogenesis. Cancer Res. 2004;64(19):7099-7109.

85. Baselga J, et al. SOLTI-0701: A multinational doubleblind, randomized phase $2 \mathrm{~b}$ study evaluating the efficacy and safety of sorafenib compared to placebo when administered in combination with capecitabine in patients with locally advanced or metastatic breast cancer (BC). Cancer Res. 2009;69(24 suppl):Abst 45.

86. Bayer. Phase III trial comparing capecitabine in combination with sorafenib or placebo in the treatment of locally advanced or metastatic HER2negative breast cancer. NIH Web site. http:// clinicaltrials.gov/ct $2 /$ show $/$ NCT01234337?term= NCT01234337\&rank=1. Accessed June 13, 2011.

87. Dave B, et al. Loss of phosphatase and tensin homolog or phosphoinositol-3 kinase activation and response to trastuzumab or lapatinib in human epidermal growth factor receptor 2-overexpressing locally advanced breast cancers. J Clin Oncol. 2011; 29(2):166-173.

88. Huober J, et al. Effect of neoadjuvant anthracyclinetaxane-based chemotherapy in different biological breast cancer phenotypes: overall results from the GeparTrio study. Breast Cancer Res Treat. 2010; 124(1):133-140

89. Gianni L, et al. Neoadjuvant chemotherapy with trastuzumab followed by adjuvant trastuzumab versus neoadjuvant chemotherapy alone, in patients with HER2-positive locally advanced breast cancer (the NOAH trial): a randomised controlled superiority trial with a parallel HER2-negative cohort. Lancet. 2010;375(9712):377-384.

90. Wolmark N, Wang J, Mamounas E, Bryant J, Fisher B. Preoperative chemotherapy in patients with operable breast cancer: nine-year results from national surgical adjuvant breast and bowel project B-18. J Natl Cancer Inst Monogr. 2001;(30):96-102.

91. Mauri D, Pavlidis N, Ioannidis JP. Neoadjuvant versus adjuvant systemic treatment in breast cancer: a meta-analysis. J Natl Cancer Inst. 2005;97(3):188-194. 